\title{
Surface Wave Propagation in a Microstretch Thermoelastic Diffusion Material under an Inviscid Liquid Layer
}

\author{
Rajneesh Kumar, ${ }^{1}$ Sanjeev Ahuja, ${ }^{2}$ and S. K. Garg ${ }^{3}$ \\ ${ }^{1}$ Department of Mathematics, Kurukshetra University, Kurukshetra, Haryana 136119, India \\ ${ }^{2}$ University Institute of Engineering \& Technology, Kurukshetra University, Kurukshetra, Haryana 136119, India \\ ${ }^{3}$ Department of Mathematics, Deen Bandhu Chotu Ram University of Science \& Technology, Sonipat, Haryana 131027, India
}

Correspondence should be addressed to Sanjeev Ahuja; sanjeev_ahuja81@hotmail.com

Received 25 February 2014; Accepted 2 July 2014; Published 4 August 2014

Academic Editor: Andrew Peplow

Copyright (C) 2014 Rajneesh Kumar et al. This is an open access article distributed under the Creative Commons Attribution License, which permits unrestricted use, distribution, and reproduction in any medium, provided the original work is properly cited.

\begin{abstract}
The present investigation deals with the propagation of Rayleigh type surface waves in an isotropic microstretch thermoelastic diffusion solid half space under a layer of inviscid liquid. The secular equation for surface waves in compact form is derived after developing the mathematical model. The dispersion curves giving the phase velocity and attenuation coefficients with wave number are plotted graphically to depict the effect of an imperfect boundary alongwith the relaxation times in a microstretch thermoelastic diffusion solid half space under a homogeneous inviscid liquid layer for thermally insulated, impermeable boundaries and isothermal, isoconcentrated boundaries, respectively. In addition, normal velocity component is also plotted in the liquid layer. Several cases of interest under different conditions are also deduced and discussed.
\end{abstract}

\section{Introduction}

A micropolar continuum is a collection of interconnected particles in the form of small rigid bodies undergoing both translational and rotational motions. Typical examples of such materials are granular media and multimolecular bodies whose microstructures act as an evident part in their macroscopic responses. Rigid chopped fibres, elastic solids with rigid granular inclusions, and other industrial materials such as liquid crystals are examples of such materials.

The theory of micropolar elasticity introduced and developed by Eringen [1] aroused much interest because of its possible utility in investigating the deformation properties of solids for which the classical theory is inadequate. The micropolar theory is believed to be particularly useful in investigating materials consisting of bar-like molecules which exhibit microrotation effects and which can support body and surface couples. Eringen [2] formulated the theory of micropolar fluids which display the effects of local rotary inertia and couple stresses. This theory can be used to explain the flow of colloidal fluids, liquid crystals, animal blood, and so forth. Eringen [3] and Nowacki [4] extended the theory of micropolar elasticity to heat conducting elastic solids by including thermal effects. Chandrasekharaiah [5] developed a heat-flux dependent generalized theory of micropolar thermoelasticity.

Eringen [6] developed the theory of micropolar elastic solid with stretch. Eringen [7] also developed the theory of microstretch thermoelastic solid. A microstretch elastic solid possesses seven degrees of freedom: three for translation, three for rotation, and one for stretch. The material points of microstretch bodies can stretch and contract independently of their rotation and translation. Composite materials reinforced with microcracks, animal bones, foams, chopped elastic fibres, porous media whose pores are filled with gas or inviscid liquid, asphalt or other elastic inclusions, "solid-liquid" crystals, and so forth fall outside the domain of micropolar elasticity; the microstretch continua can be used as mathematical models for these types of solids. A comprehensive review on the micropolar continuum theory has been given in the book by Eringen [8].

The thermodiffusion in elastic solids is due to coupling of fields of temperature, mass diffusion, and that of strain in addition to heat and mass exchange with the 
environment. Nowacki [9-12] developed the theory of thermoelastic diffusion by using coupled thermoelastic model. Dudziak and Kowalski [13] and Olesiak and Pyryev [14], respectively, discussed the theory of thermodiffusion and coupled quasistationary problems of thermal diffusion for an elastic layer. Uniqueness and reciprocity theorems for the equations of generalized thermoelastic diffusion problem, in isotropic media were proved by Sherief et al. [15]. Kumar and Kansal [16] developed the basic equation of anisotropic thermoelastic diffusion based on Green-Lindsay model.

Surface waves carry a lot of information about the Earth's crust and dispersion analysis of surface waves is concerning with the phase velocity and wave number. Further the velocity of seismic waves depends on the elastic parameters of the medium through which they travel. Therefore, many investigators have studied the surface wave propagation by considering different kinds of models. Wu and Zhu [17] studied the propagation of Lamb waves in a plate bordered with inviscid liquid layer on both sides. Zhu and $\mathrm{Wu}$ [18] derived the dispersion equations of the Lamb waves of a plate bordered with viscous liquid layer or half space viscous liquid on both sides.

Several authors discussed different problems in microstretch thermoelastic solid, notable among them are the spatial decay for the dynamic problems of thermomicrostretch elastic solid developed by Quintanilla [19]. Kumar and Partap discussed [20] the free vibration analysis of waves in a homogeneous isotropic microstretch elastic plate subjected to stress free conditions. Abbas and Othman [21] studied the propagation of plane waves in generalized thermomicrostretch elastic solid with thermal relaxation using finite element method. Othman et al. [22] studied the effect of magnetic field on the general model of equations of rotating generalized thermomicrostretch for a homogeneous isotropic elastic half-space solid whose surface is subjected to a Mode-I Crack in the context of the Green and Naghdi theory.

Propagation of Rayleigh surface waves in microstretch thermoelastic continua under inviscid fluid loadings has been investigated by Sharma et al. [23]. The propagation of free vibrations in microstretch thermoelastic homogeneous isotropic, thermally conducting plate bordered with layers of inviscid liquid on both sides subjected to stress free thermally insulated and isothermal conditions has been investigated by Kumar and Partap [24]. Recently, Kumar et al. studied the propagation of Rayleigh waves in an isotropic microstretch thermoelastic diffusion solid half-space [25].

However, no attempt has been made to discuss the effect of an imperfect boundary along with the relaxation times in a microstretch thermoelastic diffusion solid half space under a homogeneous inviscid liquid layer for thermally insulated, impermeable boundaries and isothermal, isoconcentrated boundaries, respectively.

The exact nature of the layers beneath the earth's surface is unknown. Therefore, for the purpose of theoretical investigations, one has to consider various appropriate theoretical models. The model under consideration is the motivation of the situation when inviscid liquid layer is resting on the microstretch thermoelastic diffusion medium. Since water, oil, chemicals, and so forth are present inside the earth, the propagation of surface waves in such a layer may be relevant in the exploration of oils and other valuable liquids. The problem of surface wave propagation as a part of exploration seismology is also helpful in various economical activities, like tracing of hydrocarbons and other mineral ores. The present study, in fact, is a step to attempt a more realistic model of the earth's crust and hence may be helpful in the further investigation of exploration seismology, geophysics, earthquake engineering, and soil dynamics; both theoretically and practically, we hope this study will enhance the knowledge in better understanding of the complexities of the earth medium indirectly.

In the present paper, the governing equations of isotropic microstretch thermoelastic diffusion solid half space under a layer of inviscid liquid are formulated and solved for twodimension case. The phase velocity and attenuation coefficients of the waves are studied numerically for a particular model. The study of present investigation has applications in oil companies which rely on seismic interpretation for selecting the sites for exploratory oil wells because seismic wave methods have higher precision, higher resolution, and most cost-effective technique, as compared to drilling which is expansive and time consuming.

\section{Basic Equations}

Following Eringen [8], Sherief et al. [15], and Kumar and Kansal [16], the equations of motion and the constitutive relations in a homogeneous isotropic microstretch thermoelastic diffusion solid in the absence of body forces, body couples, stretch force, and heat sources are given by

$$
\begin{aligned}
&(+2 \mu+K) \nabla(\nabla \cdot \vec{u})-(\mu+K) \nabla \times \nabla \times \vec{u} \\
&+K \nabla \times \vec{\varphi}+\lambda_{o} \nabla \varphi^{*}-\beta_{1}\left(1+\tau_{1} \frac{\partial}{\partial t}\right) \nabla T \\
&-\beta_{2}\left(1+\tau^{1} \frac{\partial}{\partial t}\right) \nabla C=\rho \frac{\partial^{2} \vec{u}}{\partial t^{2}} \\
&(\alpha+\beta+\gamma) \nabla(\nabla \cdot \vec{\varphi})-\gamma \nabla \times(\nabla \times \vec{\varphi}) \\
&+K \nabla \times \vec{u}-2 K \vec{\varphi}=\rho j \frac{\partial^{2} \vec{\varphi}}{\partial t^{2}} \\
& \alpha_{0} \nabla^{2} \varphi^{*}+v_{1}\left(T+\tau_{1} \dot{T}\right)+\nu_{2}\left(C+\tau^{1} \dot{C}\right) \\
&-\lambda_{1} \varphi^{*}-\lambda_{0} \nabla \cdot \vec{u}=\frac{\rho j_{0}}{2} \frac{\partial^{2} \varphi^{*}}{\partial t^{2}} \\
& K^{*} \nabla^{2} T=\beta_{1} T_{0}\left(1+\varepsilon \tau_{0} \frac{\partial}{\partial t}\right) \nabla \cdot \dot{\vec{u}}+\nu_{1} T_{0}\left(1+\varepsilon \tau_{0} \frac{\partial}{\partial t}\right) \dot{\varphi}^{*} \\
&+
\end{aligned}
$$


and constitutive relations are

$$
\begin{gathered}
t_{i j}=\lambda u_{r, r} \delta_{i j}+\mu\left(u_{i, j}+u_{j, i}\right)+K\left(u_{j, i}-\varepsilon_{i j r} \varphi_{r}\right) \\
+\lambda_{o} \delta_{i j} \varphi^{*}-\beta_{1}\left(1+\tau_{1} \frac{\partial}{\partial t}\right) T \delta_{i j} \\
-\beta_{2}\left(1+\tau^{1} \frac{\partial}{\partial t}\right) C \delta_{i j}, \\
m_{i j}=\alpha \varphi_{r, r} \delta_{i j}+\beta \varphi_{i, j}+\gamma \varphi_{j, i}+b_{0} \varepsilon_{m j i} \varphi_{, m}^{*}, \\
\lambda_{i}^{*}=\alpha_{0} \varphi_{, i}^{*}+b_{0} \varepsilon_{i j m} \varphi_{j, m},
\end{gathered}
$$

where $\lambda, \mu$ are Lames elastic constants, $\alpha, \beta, \gamma, K$ are micropolar constants, $\lambda_{0}, \lambda_{1}, \alpha_{o}, b_{o}$ are microstretch elastic constants, $\rho$ is the mass density, $\vec{u}=\left(u_{1}, u_{2}, u_{3}\right)$ is the displacement vector, $\vec{\varphi}=\left(\varphi_{1}, \varphi_{2}, \varphi_{3}\right)$ is the microrotation vector, $\varphi^{*}$ is the scalar microstretch function, $T$ and $T_{0}$ are the small temperature increment and the reference temperature of the body chosen such that $\left|T / T_{0}\right| \ll 1, C$ is the concentration of the diffusion material in the elastic body, $K^{*}$ is the coefficient of the thermal conductivity, $C^{*}$ the specific heat at constant strain, $D$ is the thermoelastic diffusion constant, $a, b$ are, respectively, coefficients describing the measure of thermodiffusion and of mass diffusion effects, $\beta_{1}=(3 \lambda+$ $2 \mu+K) \alpha_{t 1}, \beta_{2}=(3 \lambda+2 \mu+K) \alpha_{c 1}, \nu_{1}=(3 \lambda+2 \mu+K) \alpha_{t 2}$, $v_{2}=(3 \lambda+2 \mu+K) \alpha_{c 2}, \alpha_{t 1}, \alpha_{t 2}$ are coefficients of linear thermal expansion, and $\alpha_{c 1}, \alpha_{c 2}$ are the coefficients of linear diffusion expansion. $j$ is the microintertia, $j_{o}$ is the microinertia of the microelements, $t_{i j}$ and $m_{i j}$ are components of stress and couple stress tensors, respectively, $\lambda_{i}^{*}$ is the microstress tensor, $e_{i j}\left(=(1 / 2)\left(u_{i, j}+u_{j, i}\right)\right)$ are components of infinitesimal strain, $e_{k k}$ is the dilatation, $\delta_{i j}$ is the Kronecker delta, $\tau^{0}, \tau^{1}$ are diffusion relaxation times with $\tau^{1} \geq \tau^{0} \geq 0$, and $\tau_{0}$, $\tau_{1}$ are thermal relaxation times with $\tau_{1} \geq \tau_{0} \geq 0$. Here $\tau_{0}=\tau^{0}=\tau_{1}=\tau^{1}=\gamma_{1}=0$ for coupled thermoelastic (CT) model, $\tau_{1}=\tau^{1}=0, \varepsilon=1, \gamma_{1}=\tau_{0}$ for Lord-Shulman (L-S) model, and $\varepsilon=0, \gamma_{1}=\tau^{0}$ where $\tau^{0}>0$ for Green-Lindsay (G-L) model.

In the above equations, a comma followed by a suffix denotes spatial derivative and a superposed dot denotes the derivative with respect to time, respectively.

Following Achenbach [26], the field equations can be expressed in terms of velocity potential $\psi^{\ell}$ for inviscid fluid as

$$
\frac{\partial^{2} \psi^{\ell}}{\partial x_{1}^{2}}+\frac{\partial^{2} \psi^{\ell}}{\partial x_{3}^{2}}=\frac{1}{\left(\alpha^{\ell}\right)^{2}} \frac{\partial^{2} \psi^{\ell}}{\partial t^{2}},
$$

where $\alpha^{\ell}=\sqrt{\lambda^{\ell} / \rho^{\ell}}$ is the velocity of acoustic fluid, $\lambda^{\ell}$ is the bulk modulus, and $\rho^{\ell}$ is the density of the fluid.

The velocity components $u_{1}^{\ell}, u_{3}^{\ell}$ and the acoustic pressure $p^{\ell}$ in the medium $M_{2}$ are given by

$$
u_{1}^{\ell}=\frac{\partial \psi^{\ell}}{\partial x_{1}}, \quad u_{3}^{\ell}=\frac{\partial \psi^{\ell}}{\partial x_{3}}, \quad p^{\ell}=-\rho^{\ell} \frac{\partial \psi^{\ell}}{\partial t} .
$$

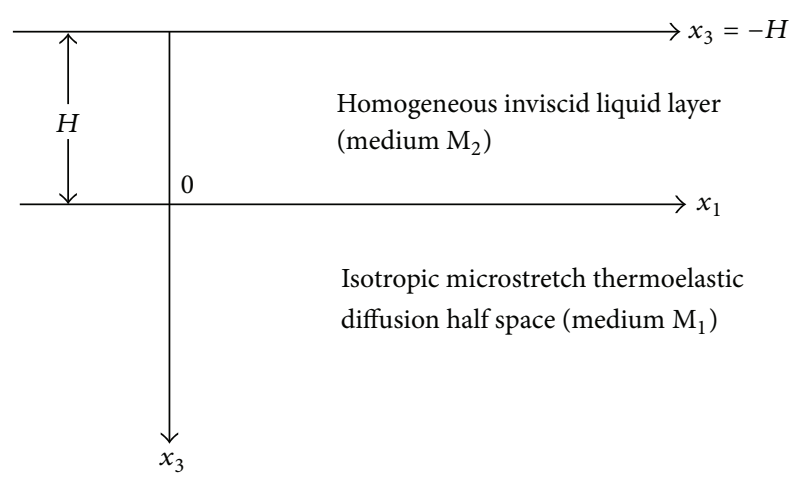

FIGURE 1: Geometry of the problem.

\section{Formulation of the Problem}

We consider a homogeneous, isotropic microstretch thermoelastic diffusion medium $\left(M_{1}\right)$ lying under a uniform homogeneous inviscid liquid layer medium $\left(M_{2}\right)$ of thickness $H$ (Figure 1). We take the rectangular Cartesian coordinate system $O x_{1} x_{2} x_{3}$ at any point on the plane horizontal surface and $x_{1}$-axis in the direction of wave propagation and $x_{3}$-axis pointing vertically downward into the half-space so that all particles on a line parallel to $x_{2}$-axis are equally displaced; therefore, all the field quantities will be independent of $x_{2}$ coordinates. Hence the liquid layer medium $M_{2}$ occupies the region $-H<x_{3}<0$ and $x_{3}>0$ is occupied by the half-space medium $M_{1}$. The plane $x_{3}=-H$ represents the free surface of the liquid and $x_{3}=0$ is taken as interface between $M_{1}$ and $M_{2}$ medium. For the two-dimensional problem, we take

$$
\begin{gathered}
\vec{u}\left(x_{1}, x_{3}, t\right)=\left(u_{1}, 0, u_{3}\right), \\
\vec{\varphi}=\left(0, \varphi_{2}, 0\right), \varphi^{*}\left(x_{1}, x_{3}, t\right), T\left(x_{1}, x_{3}, t\right), C\left(x_{1}, x_{3}, t\right) .
\end{gathered}
$$

We define the following dimensionless quantities:

$$
\begin{gathered}
\left(x_{1}^{\prime}, x_{3}^{\prime}\right)=\frac{\omega^{*}}{c_{1}}\left(x_{1}, x_{3}\right), \quad\left(u_{1}^{\prime}, u_{3}^{\prime}\right)=\frac{\rho c_{1} \omega^{*}}{\beta_{1} T_{o}}\left(u_{1}, u_{3}\right), \\
\varphi_{2}^{\prime}=\frac{\rho c_{1}^{2}}{\beta_{1} T_{o}} \varphi_{2}, \quad \varphi^{* \prime}=\frac{\rho c_{1}^{2}}{\beta_{1} T_{o}} \varphi^{*}, \quad t_{i j}^{\prime}=\frac{t_{i j}}{\beta_{1} T_{o}}, \\
m_{i j}^{\prime}=\frac{\omega^{*}}{c_{1} \beta_{1} T_{o}} m_{i j}, \quad \lambda_{i}^{* \prime}=\frac{\lambda_{i}^{*} \omega^{*}}{c_{1} \beta_{1} T_{o}}, \quad T^{\prime}=\frac{T}{T_{o}}, \\
C^{\prime}=\frac{\beta_{2} C}{\rho c_{1}^{2}}, \quad t^{\prime}=\omega^{*} t, \quad \tau_{o}^{\prime}=\omega^{*} \tau_{o}, \quad \tau^{0 \prime}=\omega^{*} \tau^{0}, \\
\tau_{1}^{\prime}=\omega^{*} \tau_{1}, \quad \tau^{1 \prime}=\omega^{*} \tau^{1}, \quad h^{\prime}=\frac{c_{1}}{\omega^{*}} h,
\end{gathered}
$$


and

$$
\begin{gathered}
\left(u_{1}^{\prime}, u_{3}^{\prime \ell}\right)=\frac{1}{c_{1}}\left(u_{1}^{\ell}, u_{3}^{\ell}\right), \quad p^{\ell^{\prime}}=\frac{p^{\ell}}{\mu}, \quad \psi^{\ell^{\prime}}=\frac{\omega^{*} \psi^{\ell}}{c_{1}^{2}} \\
K_{n}^{\prime}=\frac{c_{1}}{\mu} K_{n}
\end{gathered}
$$

where $\omega^{*}=\rho C^{*} c_{1}^{2} / K^{*}, c_{1}^{2}=(\lambda+2 \mu+K) / \rho, \omega^{*}$ is the characteristic frequency of the medium.

Upon introducing the quantities (6) and (7) in (1), with the aid of (5) and after suppressing the primes, we obtain

$$
\begin{gathered}
\delta^{2} \frac{\partial e}{\partial x_{1}}+\left(1-\delta^{2}\right) \nabla^{2} u_{1}-a_{1} \frac{\partial \varphi_{2}}{\partial x_{3}}+a_{2} \frac{\partial \varphi^{*}}{\partial x_{1}}-\tau_{t}^{1} \frac{\partial T}{\partial x_{1}} \\
-a_{3} \tau_{c}^{1} \frac{\partial C}{\partial x_{1}}=\frac{\partial^{2} u_{1}}{\partial t^{2}} \\
\delta^{2} \frac{\partial e}{\partial x_{3}}+\left(1-\delta^{2}\right) \nabla^{2} u_{3}+a_{1} \frac{\partial \varphi_{2}}{\partial x_{1}}+a_{2} \frac{\partial \varphi^{*}}{\partial x_{3}}-\tau_{t}^{1} \frac{\partial T}{\partial x_{3}} \\
-a_{3} \tau_{c}^{1} \frac{\partial C}{\partial x_{3}}=\frac{\partial^{2} u_{3}}{\partial t^{2}}, \\
a_{4} \nabla^{2} \varphi_{2}+a_{5}\left(\frac{\partial u_{1}}{\partial x_{3}}-\frac{\partial u_{3}}{\partial x_{1}}\right)-a_{6} \varphi_{2}=\frac{\partial^{2} \varphi_{2}}{\partial t^{2}} \\
\left(\delta_{1}^{2} \nabla^{2}-a_{7}\right) \varphi^{*}-a_{8} e+a_{9} \tau_{t}^{1} T+a_{10} \tau_{c}^{1} C=\frac{\partial^{2} \varphi^{*}}{\partial t^{2}} \\
\nabla^{2} T=a_{11} \tau_{e}^{0} \frac{\partial e}{\partial t}+a_{12} \tau_{e}^{0} \frac{\partial \varphi^{*}}{\partial t}+\tau_{t}^{0} \frac{\partial T}{\partial t}+a_{13} \tau_{c}^{0} \frac{\partial C}{\partial t} \\
\nabla^{2} e+a_{21} \nabla^{2} \varphi^{*}+a_{15} \tau_{t}^{1} \nabla^{2} T+\tau_{f}^{0} \frac{\partial C}{\partial t}-a_{16} \tau_{c}^{1} \nabla^{2} C=0
\end{gathered}
$$

where

$$
\begin{gathered}
\left(a_{1}, a_{2}\right)=\frac{1}{\rho c_{1}^{2}}\left(K, \lambda_{0}\right), \quad a_{3}=\frac{\rho c_{1}^{2}}{\beta_{1} T_{0}}, \\
\left(a_{4}, a_{5}, a_{6}\right)=\frac{1}{j \rho}\left(\frac{\gamma}{c_{1}^{2}}, \frac{K}{\omega^{* 2}}, \frac{2 K}{\omega^{* 2}}\right), \quad \delta^{2}=\frac{\lambda+\mu}{\rho c_{1}^{2}} \\
\left(a_{11}, a_{12}, a_{13}\right)=\frac{1}{K^{*} \omega^{*}}\left(\frac{T_{0} \beta_{1}^{2}}{\rho}, \frac{\beta_{1} T_{0} \nu_{1}}{\rho}, \frac{\rho c_{1}^{4} a}{\beta_{2}}\right) \\
\left(a_{14}, a_{15}, a_{16}\right)=\frac{D \omega^{*}}{c_{1}^{2}}\left(\frac{\beta_{1}^{2}}{\rho c_{1}^{2}}, \frac{\beta_{2} a}{\beta_{1}}, b\right) \\
\left(a_{7}, a_{8}, a_{9}, a_{10}\right)=\frac{2}{j_{0} \omega^{* 2}}\left(\frac{\lambda_{1}}{\rho}, \frac{\lambda_{0}}{\rho}, \frac{\nu_{1} c_{1}^{2}}{\beta_{1}}, \frac{\nu_{2} \rho c_{1}^{4}}{\beta_{1} \beta_{2} T_{0}}\right) \\
\delta_{1}^{2}=\frac{c_{2}^{2}}{c_{1}^{2}}, \quad c_{2}^{2}=\frac{2 \alpha_{0}}{\rho j_{0}}, \quad a_{21}=\frac{D \nu \beta_{2} \omega^{*}}{\rho c_{1}^{4}} \\
\tau_{t}^{1}=1+\tau_{1} \frac{\partial}{\partial t}, \quad \tau_{c}^{1}=1+\tau^{1} \frac{\partial}{\partial t}, \\
\tau_{f}^{0}=1+\varepsilon \tau^{0} \frac{\partial}{\partial t}, \quad \tau_{t}^{0}=1+\tau_{0} \frac{\partial}{\partial t}
\end{gathered}
$$

$$
\begin{array}{cc}
\tau_{e}^{0}=1+\varepsilon \tau_{0} \frac{\partial}{\partial t}, & \tau_{c}^{0}=1+\gamma_{1} \frac{\partial}{\partial t} \\
e=\frac{\partial u_{1}}{\partial x_{1}}+\frac{\partial u_{3}}{\partial x_{3}}, & \nabla^{2}=\frac{\partial^{2}}{\partial x_{1}^{2}}+\frac{\partial^{2}}{\partial x_{3}^{2}}
\end{array}
$$

We introduce the potential functions $\phi$ and $\psi$ through the relations

$$
u_{1}=\frac{\partial \phi}{\partial x_{1}}-\frac{\partial \psi}{\partial x_{3}}, \quad u_{3}=\frac{\partial \phi}{\partial x_{3}}+\frac{\partial \psi}{\partial x_{1}} .
$$

In (8); we obtain

$$
\begin{gathered}
\nabla^{2} \phi+a_{2} \varphi^{*}-\tau_{t}^{1} T-a_{3} \tau_{c}^{1} C=\ddot{\phi}, \\
\left(1-\delta^{2}\right) \nabla^{2} \psi+a_{1} \varphi_{2}=\ddot{\psi}, \\
\left(a_{4} \nabla^{2}-a_{6}\right) \varphi_{2}-a_{5} \nabla^{2} \psi=\ddot{\varphi}_{2}, \\
\left(\delta_{1}^{2} \nabla^{2}-a_{7}\right) \varphi^{*}-a_{8} \nabla^{2} \phi+a_{9} \tau_{t}^{1} T+a_{10} \tau_{c}^{1} C=\ddot{\varphi}^{*}, \\
\nabla^{2} T=\tau_{e}^{0}\left(a_{11} \nabla^{2} \dot{\phi}+a_{12} \dot{\varphi}^{*}\right)+\tau_{t}^{0} \dot{T}+a_{13} \tau_{c}^{0} \dot{C}, \\
a_{14} \nabla^{4} \phi+a_{21} \nabla^{2} \varphi^{*}+a_{15} \tau_{t}^{1} \nabla^{2} T-a_{16} \tau_{c}^{1} \nabla^{2} C+\tau_{f}^{0} \dot{C}=0 .
\end{gathered}
$$

In order to solve (11)-(16), we assume

$$
\begin{aligned}
\left\{\phi, \varphi^{*}, T, C, \psi^{\ell}\right\}\left(x_{1}, x_{3}, t\right) & =\left\{\bar{\phi}, \bar{\varphi}^{*}, \bar{T}, \bar{C}, \bar{\psi}^{\ell}\right\}\left(x_{3}\right) e^{i \xi\left(x_{1}-c t\right)}, \\
\left\{\psi, \varphi_{2}\right\}\left(x_{1}, x_{3}, t\right) & =\left\{\bar{\psi}, \bar{\varphi}_{2}\right\}\left(x_{3}\right) e^{i \xi\left(x_{1}-c t\right)}
\end{aligned}
$$

where $\xi$ is the wave number, $\omega=\xi c$ is the angular frequency, and $c$ is phase velocity of the wave. Using (17) in (11) and (14)-(16), we obtain a system of four homogeneous equations in four unknowns $\bar{\phi}, \bar{\varphi}^{*}, \bar{T}$, and $\bar{C}$ which for the nontrivial solution yields

$$
D^{8}+A_{1}^{*} D^{6}+B_{1}^{*} D^{4}+C_{1}^{*} D^{2}+D_{1}^{*}=0
$$

and using (18) in (12) and (13), we obtain a system of two homogeneous equations in two unknowns $\bar{\psi}$ and $\bar{\varphi}_{2}$ which for the nontrivial solution yields

$$
D^{4}+A_{2}^{*} D^{2}+B_{2}^{*}=0
$$

where $D=d / d x_{3}$, and the coefficients $A_{1}^{*}, B_{1}^{*}, C_{1}^{*}, D_{1}^{*}, A_{2}^{*}$, and $B_{2}^{*}$ are given in Appendix $A$.

Let the roots of (19) be denoted by $m_{p}^{2}(p=1,2,3,4)$. Four positive values of $c$ in the descending order will be the velocities of propagation of four possible waves, namely, longitudinal displacement wave (LD), thermal wave (T), mass diffusion wave (MD), and longitudinal microstretch wave (LM), respectively. Similarly, for two roots of (20) denoted by $m_{p}^{2}(p=5,6)$, the positive values of $c$ in the descending order will be the velocities of propagation of two coupled transverse displacement and transverse microrotational waves (CD I, CD II), respectively. Since we are interested in surface waves 
only, it is essential that the motion is confined to the free surface of $x_{3}=0$ in the half-space so that the characteristic roots satisfy the radiation conditions $\operatorname{Re}\left(m_{p}\right) \geq 0,(p=$ $1,2,3,4,5,6)$. Thus, the solution of field equations takes the form

$$
\begin{gathered}
\left\{\phi, \varphi^{*}, T, C\right\}=\sum_{p=1}^{4}\left[A_{p}\left\{1, n_{1 p}, n_{2 p}, n_{3 p}\right\} e^{-m_{p} x_{3}}\right] e^{i \xi\left(x_{1}-c t\right)}, \\
\left\{\psi, \varphi_{2}\right\}=\sum_{p=5}^{6}\left[A_{p}\left\{1, n_{4 p}\right\} e^{-m_{p} x_{3}}\right] e^{i \xi\left(x_{1}-c t\right)},
\end{gathered}
$$

where $A_{p}(p=1,2,3,4,5,6)$ are arbitrary constants.

The coupling constants $n_{1 p}, n_{2 p}, n_{3 p}$, and $n_{4 p}$ are given in Appendix B.

Substituting the values of $\psi^{\ell}$ from (17) in (3) and after simplification, we obtain

$$
\psi^{\ell}=\left[A_{7} \cos \left(m_{7} x_{3}\right)+A_{8} \sin \left(m_{7} x_{3}\right)\right] \exp \left(i \xi\left(x_{1}-c t\right)\right),
$$

where

$$
m_{7}= \pm \xi\left(\frac{c_{1}^{2} c^{2}}{\left(\alpha^{\ell}\right)^{2}}-1\right)^{1 / 2}
$$

and $A_{7}, A_{8}$ are arbitrary constants.

The velocity component and acoustic pressure for medium $M_{2}$, with the help of (3), (4), (7), and (22), are given by

$$
\begin{aligned}
u_{3}^{\ell}= & m_{7}\left[-A_{7} \sin \left(m_{7} x_{3}\right)+A_{8} \cos \left(m_{7} x_{3}\right)\right] \\
& \times \exp \left(i \xi\left(x_{1}-c t\right)\right), \\
p^{\ell}= & \varepsilon^{\ell}\left[A_{7} \cos \left(m_{7} x_{3}\right)+A_{8} \sin \left(m_{7} x_{3}\right)\right] \\
& \times \exp \left(i \xi\left(x_{1}-c t\right)\right),
\end{aligned}
$$

where $\varepsilon^{\ell}=i \rho^{\ell} c_{1}^{2} c \xi / \mu$.

\section{Boundary Conditions}

The appropriate boundary conditions are as follows.

(i) Vanishing of the pressure at the free surface of the liquid layer, $x_{3}=-H$; that is,

$$
p^{\ell}=0 \text {. }
$$

(ii) Continuity of normal stress components at the interface $x_{3}=0$; that is,

$$
\left(t_{33}\right)_{M_{1}}=-p^{\ell}
$$

(iii) Discontinuity of the pressure at the interface $x_{3}=0$; that is,

$$
p^{\ell}=K_{n}\left(\left(\dot{u}_{3}\right)_{M_{1}}-\left(u_{3}\right)_{M_{2}}\right) .
$$

(iv) Vanishing of shear stress for medium $M_{1}$ at the interface $x_{3}=0$; that is,

$$
\left(t_{31}\right)_{M_{1}}=0
$$

(v) Vanishing of the tangential couple stress at the interface $x_{3}=0$; that is,

$$
\left(m_{32}\right)_{M_{1}}=0
$$

(vi) Vanishing of the normal microstress component at the interface $x_{3}=0$; that is,

$$
\left(\lambda_{3}^{*}\right)_{M_{1}}=0
$$

(vii) Thermal boundary conditions

$$
\frac{\partial T}{\partial x_{3}}+h_{1} T=0
$$

$$
\text { where } \begin{cases}h_{1} \longrightarrow 0 & \begin{array}{l}
\text { corresponds Insulated } \\
\text { boundary condition }
\end{array} \\
h_{1} \longrightarrow \infty & \begin{array}{l}
\text { corresponds Isolated } \\
\text { boundary condition. }
\end{array}\end{cases}
$$

(viii) Consider

$$
\begin{aligned}
& \frac{\partial C}{\partial x_{3}}+h_{2} C=0, \\
& \text { where } \begin{cases}h_{2} \longrightarrow 0 & \begin{array}{l}
\text { corresponds to impermeable } \\
\text { boundary }
\end{array} \\
h_{2} \longrightarrow \infty & \begin{array}{l}
\text { refers to isoconcentrated } \\
\text { boundary }
\end{array}\end{cases}
\end{aligned}
$$

where

$$
\begin{gathered}
t_{33}=\frac{\partial u_{3}}{\partial x_{3}}+b_{1} \frac{\partial u_{1}}{\partial x_{1}}-\tau_{t}^{1} T-\tau_{c}^{1} C+a_{2} \varphi^{*} \\
t_{31}=b_{2} \frac{\partial u_{1}}{\partial x_{3}}+b_{3} \frac{\partial u_{3}}{\partial x_{1}}-a_{1} \varphi_{2}, \quad m_{32}=b_{4} \frac{\partial \varphi_{2}}{\partial x_{3}}+b_{5} \frac{\partial \varphi^{*}}{\partial x_{1}} \\
\lambda_{3}^{*}=b_{6} \frac{\partial \varphi^{*}}{\partial x_{3}}-b_{5} \frac{\partial \varphi_{2}}{\partial x_{1}} \\
\left(b_{1}, b_{2}, b_{3}\right)=\frac{1}{\rho c_{1}^{2}}(\lambda, \mu+K, \mu) \\
\left(b_{4}, b_{5}, b_{6}\right)=\frac{\omega^{* 2}}{\rho c_{1}^{4}}\left(\gamma, b_{o}, \alpha_{o}\right) .
\end{gathered}
$$




\section{Derivations of the Secular Equations}

Making use of (21) and (24) in the (25)-(32), we obtain a system of eight simultaneous linear equations:

$$
\begin{gathered}
\sum_{p=1}^{8} k_{1 p} A_{p}=0, \quad \sum_{p=1}^{8} k_{2 p} A_{p}=0, \quad \sum_{p=1}^{8} k_{3 p} A_{p}=0, \\
\sum_{p=1}^{8} k_{4 p} A_{p}=0, \quad \sum_{p=1}^{8} k_{5 p} A_{p}=0, \quad \sum_{p=1}^{8} k_{6 p} A_{p}=0, \\
\sum_{p=1}^{8} k_{7 p} A_{p}=0, \quad \sum_{p=1}^{8} k_{8 p} A_{p}=0,
\end{gathered}
$$

where

$$
\begin{aligned}
& k_{1 p}= \begin{cases}0, & \text { for }(p=1,2,3,4,5,6) \\
\cos \left(m_{7} H\right), & \text { for }(p=7) \\
-\sin \left(m_{7} H\right), & \text { for }(p=8)\end{cases} \\
& k_{2 p}=\left\{\begin{aligned}
m_{p}^{2}-b_{1} \xi^{2}+a_{2} n_{1 p} & \\
+n_{2 p}\left(i \xi_{c} \tau_{1}-1\right) & \\
+n_{3 p}\left(i \xi c \tau^{1}-1\right), & \text { for }(p=1,2,3,4), \\
i \xi m_{p}\left(b_{1}-1\right), & \text { for }(p=5,6), \\
\varepsilon^{\ell}, & \text { for }(p=7), \\
0, & \text { for }(p=8),
\end{aligned}\right. \\
& k_{3 p}= \begin{cases}\frac{i \xi_{c} K_{n} m_{p}}{a_{3}}, & \text { for }(p=1,2,3,4), \\
\frac{K_{n} \xi^{2} c}{a_{3}}, & \text { for }(p=5,6), \\
\varepsilon^{\ell^{2},} & \text { for }(p=7), \\
-K_{n} m_{7}, & \text { for }(p=8)\end{cases} \\
& k_{4 p}= \begin{cases}-i \xi m_{p}\left(b_{2}+b_{3}\right), & \text { for }(p=1,2,3,4), \\
-\left(b_{2} m_{p}^{2}+b_{3} \xi^{2}+a_{1} n_{4 p}\right), & \text { for }(p=5,6), \\
0, & \text { for }(p=7,8),\end{cases} \\
& k_{5 p}= \begin{cases}i \xi b_{5} n_{1 p}, & \text { for }(p=1,2,3,4), \\
-b_{4} n_{4 p} m_{p}, & \text { for }(p=5,6), \\
0, & \text { for }(p=7,8),\end{cases} \\
& k_{6 p}= \begin{cases}n_{1 p} m_{p} b_{6}, & \text { for }(p=1,2,3,4), \\
i \xi b_{5} n_{4 p}, & \text { for }(p=5,6), \\
0, & \text { for }(p=7,8),\end{cases} \\
& k_{7 p}= \begin{cases}\left(h_{1}-m_{p}\right) n_{2 p}, & \text { for }(p=1,2,3,4), \\
0, & \text { for }(p=5,6,7,8),\end{cases} \\
& k_{8 p}= \begin{cases}\left(h_{2}-m_{p}\right) n_{3 p}, & \text { for }(p=1,2,3,4), \\
0, & \text { for }(p=5,6,7,8) .\end{cases}
\end{aligned}
$$

The system of (34) has a nontrivial solution if the determinant of amplitudes $A_{P}, \quad(p=1,2,3,4,5,6,7,8)$ vanishes which leads to the secular equation

$$
\left|k_{i j}\right|_{8 \times 8}=0, \quad(i, j=1,2,3,4,5,6,7,8)
$$

which can further be simplified as

$$
c^{2}=\left(\frac{\alpha^{\ell}}{c_{1}}\right)^{2}\left(\left[\frac{1}{\xi H} \tan ^{-1}\left(\Delta_{12}\right)\right]^{2}+1\right) \text {, }
$$

where $\Delta_{12}$ is given in Appendix B.

Equation (37) is Rayleigh type surface wave secular equation of an isotropic microstretch thermoelastic diffusion solid half-space under a uniform homogeneous inviscid liquid layer. The secular equation has complete information about the phase velocity, attenuation coefficient, and wave number of surface waves in such medium.

\section{Particular Cases}

(i) If we take, $K_{n} \rightarrow \infty$ in (37), we obtained the frequency equation at the welded contact between an inviscid liquid layer and an isotropic microstretch thermoelastic diffusion solid half space with the changed values of $k_{i j}$ under

$$
\begin{gathered}
k_{3 p}=\frac{i \xi c m_{p}}{a_{3}}, \quad \text { for }(p=1,2,3,4), \\
k_{3 p}=\frac{\xi^{2} c}{a_{3}}, \quad \text { for }(p=5,6), \\
k_{3 p}=0, \quad \text { for }(p=7), \\
k_{3 p}=-m_{7}, \quad \text { for }(p=8) .
\end{gathered}
$$

(ii) Taking $H \rightarrow 0$ and $K_{n} \rightarrow \infty$, in secular equation (37), we obtained the frequency equation for Rayleigh waves propagating along the free surface of a microstretch thermoelastic diffusion solid half space as

$$
\left|\begin{array}{llllll}
k_{21} & k_{22} & k_{23} & k_{24} & k_{25} & k_{26} \\
k_{41} & k_{42} & k_{43} & k_{44} & k_{45} & k_{46} \\
k_{51} & k_{52} & k_{53} & k_{54} & k_{55} & k_{56} \\
k_{61} & k_{62} & k_{63} & k_{64} & k_{65} & k_{66} \\
k_{71} & k_{72} & k_{73} & k_{74} & k_{75} & k_{76} \\
k_{81} & k_{82} & k_{83} & k_{84} & k_{85} & k_{86}
\end{array}\right|_{6 \times 6}
$$

with the changed values of $k_{i j}$ in (37), that is, $k_{3 p}=$ 0 , for $p=1,2, \ldots, 6 ; k_{q 7}=k_{q 8}=0$, for $q=$ $2,3, \ldots, 8$. The frequency equation (39) in reduced form is similar as that obtained by Kumar et al. [25].

(iii) In the absence of microstretch effect, that is, taking $j_{0}=\alpha_{0}=b_{0}=\lambda_{0}=\lambda_{1}=\nu_{1}=\nu_{2}=0$ in (37), we obtained the frequency equation at the imperfect boundary between inviscid liquid layer and 
a micropolar thermoelastic diffusion solid half space as

$$
\tan \left(m_{7} H\right)=-\frac{\Delta_{3}}{\Delta_{4}}
$$

where the values of $\Delta_{3}$ and $\Delta_{4}$ are given in Appendix B and with the changed values of $k_{i j}$ in (37) as

$$
\begin{aligned}
& k_{i 4}=0, \quad \text { for } i=1,2, \ldots, 8, \\
& k_{5 j}=0, \quad \text { for } j=1,2, \ldots, 4, \\
& k_{6 j}=0, \quad \text { for } j=1,2, \ldots, 6 .
\end{aligned}
$$

(iv) In the absence of microstretch and micropolarity effects, that is, taking $j_{0}=\alpha_{0}=b_{0}=\lambda_{0}=\lambda_{1}=\nu_{1}=$ $\nu_{2}=0$ and $K=\gamma=0$ in (37), the frequency equation at an imperfect boundary between inviscid liquid layer and thermoelastic diffusion solid half space is obtained as

$$
\tan \left(m_{7} H\right)=-\frac{\Delta_{5}}{\Delta_{6}},
$$

where the values of $\Delta_{5}$ and $\Delta_{6}$ are given in Appendix B and with the changed values of $k_{i j}$ in (37) as

$$
\begin{aligned}
& k_{i 4}=0, \quad \text { for } i=1,2, \ldots, 8, \\
& k_{5 j}=0, \quad \text { for } j=1,2, \ldots, 6, \\
& k_{6 j}=0, \quad \text { for } j=1,2, \ldots, 6 .
\end{aligned}
$$

(v) In the absence of diffusion effect and $K_{n} \rightarrow \infty$, the frequency equation (37) reduced to the microstretch thermoelastic solid half space lying under inviscid liquid. The resulting frequency equation in reduced form is similar as that obtained by Sharma et al. [23].

(vi) If we take $h_{1} \rightarrow 0$ and $h_{2} \rightarrow 0$ in (37), we obtained the frequency equation for the thermally insulated and impermeable boundary with imperfect interface between inviscid liquid layer and a microstretch thermoelastic diffusion solid half space.

(vii) If we take $h_{1} \rightarrow \infty$ and $h_{2} \rightarrow \infty$ in (37), we obtained the frequency equation for the thermally insolated and isoconcentrated boundary with imperfect interface between inviscid liquid layer and a microstretch thermoelastic diffusion solid half space.

\section{Numerical Results and Discussion}

The analysis is conducted for a magnesium crystal-like material. Following Eringen [27], the values of micropolar parameters are

$$
\begin{array}{cc}
\lambda=9.4 \times 10^{10} \mathrm{Nm}^{-2}, & \mu=4.0 \times 10^{10} \mathrm{Nm}^{-2}, \\
K=1.0 \times 10^{10} \mathrm{Nm}^{-2}, & \rho=1.74 \times 10^{3} \mathrm{Kgm}^{-3}, \\
j=0.2 \times 10^{-19} \mathrm{~m}^{-2}, & \gamma=0.779 \times 10^{-9} \mathrm{~N} .
\end{array}
$$

Thermal and diffusion parameters are given by

$$
\begin{array}{cc}
C^{*}=1.04 \times 10^{3} \mathrm{JKg}^{-1} \mathrm{~K}^{-1}, & \mathrm{~K}^{*}=1.7 \times 10^{6} \mathrm{Jm}^{-1} \mathrm{~s}^{-1} \mathrm{~K}^{-1}, \\
\alpha_{t 1}=2.33 \times 10^{-5} \mathrm{~K}^{-1}, & \alpha_{t 2}=2.48 \times 10^{-5} \mathrm{~K}^{-1}, \\
T_{0}=0.298 \times 10^{3} \mathrm{~K}, & \tau_{1}=0.01, \quad \tau_{0}=0.02, \\
\alpha_{c 1}=2.65 \times 10^{-4} \mathrm{~m}^{3} \mathrm{Kg}^{-1}, & \alpha_{c 2}=2.83 \times 10^{-4} \mathrm{~m}^{3} \mathrm{Kg}^{-1}, \\
a=2.9 \times 10^{4} \mathrm{~m}^{2} \mathrm{~s}^{-2} \mathrm{~K}^{-1}, & b=32 \times 10^{5} \mathrm{Kg}^{-1} \mathrm{~m}^{5} \mathrm{~s}^{-2}, \\
\tau^{1}=0.04, \quad \tau^{0}=0.03, & D=0.85 \times 10^{-8} \mathrm{Kgm}^{-3} \mathrm{~s}, \\
H=1 .
\end{array}
$$

The microstretch parameters are taken as

$$
\begin{array}{cc}
j_{o}=0.19 \times 10^{-19} \mathrm{~m}^{2}, & \alpha_{o}=0.779 \times 10^{-9} \mathrm{~N}, \\
b_{o}=0.5 \times 10^{-9} \mathrm{~N}, & \lambda_{o}=0.5 \times 10^{10} \mathrm{Nm}^{-2}, \\
\lambda_{o}=0.5 \times 10^{10} \mathrm{Nm}^{-2}, & \lambda_{1}=0.5 \times 10^{10} \mathrm{Nm}^{-2} .
\end{array}
$$

The physical constants for water are given by $\rho^{\ell}=1.0 \times$ $10^{3} \mathrm{Kg} / \mathrm{m}^{3}, \lambda^{\ell}=2.1904 \times 10^{3} \mathrm{~N} / \mathrm{m}^{2}$, and $K_{n}=1.4 \mathrm{Nsm}^{-3}$.

MATLAB software 7.04 has been used for numerical computation of the resulting quantities. The given cost for solving the dispersion relation for a given wavenumber is equivalent to solving a system of two independent quadratic equations; in MATLAB the computational efforts is negligible; the CPU cost is also independent of the input parameter set. The values of the phase velocity and attenuation coefficients with wave number are plotted graphically to depict the effect of an imperfect boundary along with the relaxation times in a microstretch thermoelastic diffusion solid half space under a homogeneous inviscid liquid layer for thermally insulated, impermeable boundaries and isothermal, isoconcentrated boundaries, respectively. Normal velocity component has also been determined in the liquid medium with $H=1$ for the above cases and is shown in Figures 2, 3, 4, 5, 6, and 7 , respectively. For the thermally insulated and impermeable boundaries, the words LSH10IB and GLH10IB symbolize the graphs for L-S and G-L theories at an imperfect boundary between an inviscid liquid layer and a microstretch thermoelastic diffusion half space, whereas the words LSH10WC and GLH10WC symbolize the graphs for L-S and G-L theories at a welded contact between an inviscid liquid layer and a microstretch thermoelastic diffusion half space, respectively. Similarly, for the isothermal, isoconcentrated boundaries, the words LSH1NIB and GLH1NIB symbolize the graphs for L-S and G-L theories at an imperfect boundary between an inviscid liquid layer and a microstretch thermoelastic diffusion half space, whereas the words LSH1NWC and GLH1NWC symbolize the graphs for L-S and G-L theories at a welded contact between an inviscid liquid layer and a microstretch thermoelastic diffusion half space, respectively. 


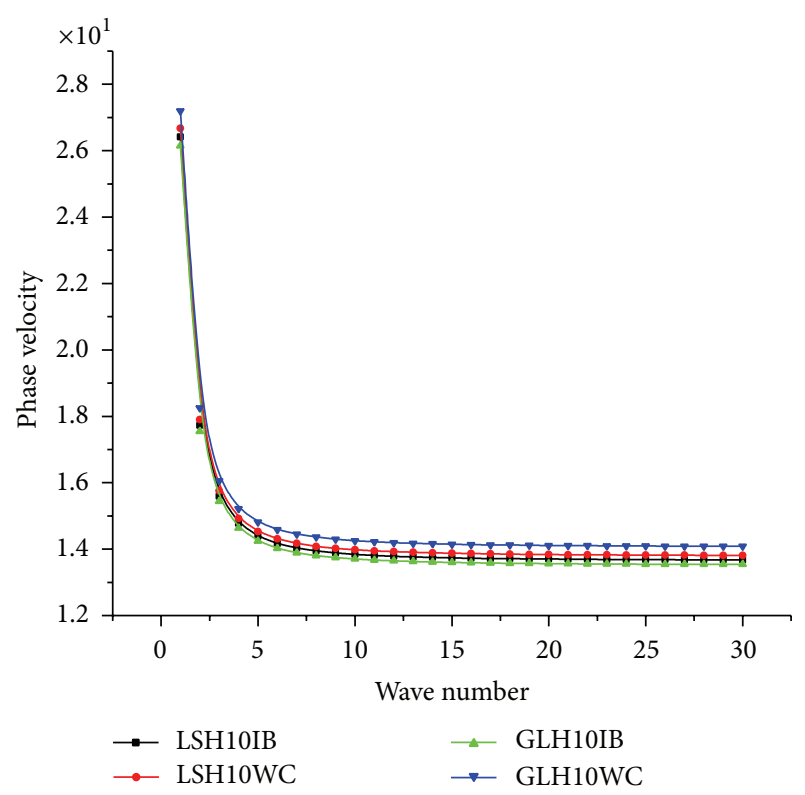

FIGURE 2: Variation of phase velocity with respect to wave number for thermally insulated and impermeable boundaries.

7.1. Phase Velocity, Attenuation Coefficient, and the Normal Velocity Component at the Thermally Insulated and Impermeable Boundaries. Figure 2 depicts the variation of phase velocity with wave number $\xi$. It is noticed that, for smaller values of $\xi$, the values of phase velocity for LSH10IB and LSH10WC decrease monotonically, whereas for higher values of $\xi$, the values of phase velocity decrease smoothly and finally become dispersionless; nevertheless, the trend of variation and behavior of the phase velocity for GLH10IB and GLH10WC is similar to LSH10IB and LSH10WC, respectively; however, the corresponding values are different in magnitude.

Figure 3 exhibits the variation of attenuation with wave number $\xi$. The values of attenuation for smaller values of wave number increase sharply for all the cases and become oscillatory for LSH10IB and LSH10WC in $4 \leq \xi \leq 6$ and instead increase gradually for GLH10IB and GLH10WC, which becomes stationary for higher values of $\xi$ for all the cases. A notable effect of relaxation times is also observed on the propagation of Rayleigh type surface waves at the imperfect boundary for $\xi=4$. Figure 4 exhibits the variation of normal velocity component $u_{3}^{\ell}$ with wave number $\xi$. The trend of variation and behavior of $u_{3}^{\ell}$ for all the cases is similar in the range $1 \leq \xi \leq 15$; nevertheless, due to imperfect boundary, a monotonic decrease in the values of $u_{3}^{\ell}$ is noticed for GLH10IB while compared with GLH10WC within the range $16 \leq \xi \leq 24$.

7.2. Phase Velocity, Attenuation Coefficient, and the Normal Velocity Component at Isothermal and Isoconcentrated Boundaries. Figure 5 depicts the variation of phase velocity with wave number $\xi$. It is noticed that the trend of variation and behavior of the phase velocity at the isothermal and isoconcentrated boundaries is similar to Figure 2, respectively,

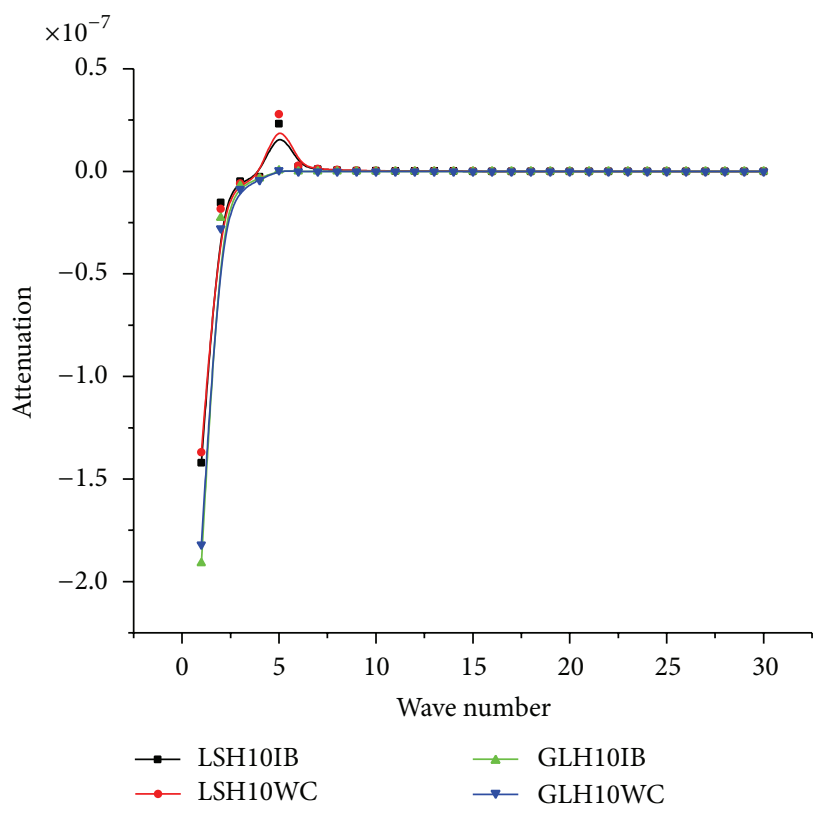

FIGURE 3: Variation of attenuation with respect to wave number for thermally insulated and impermeable boundaries.

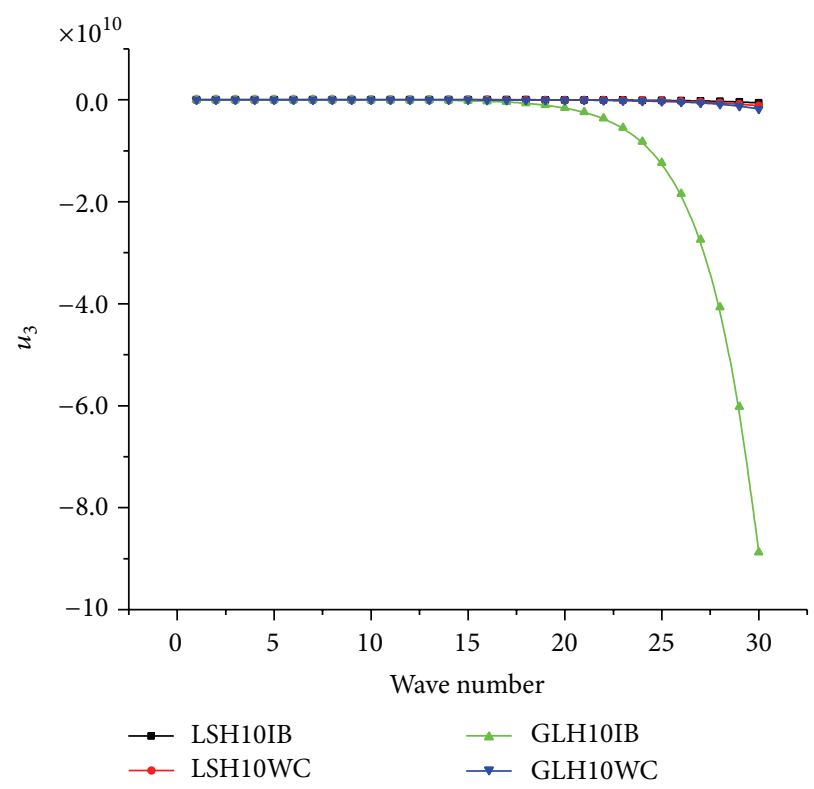

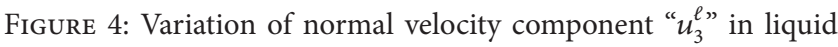
half-space with respect to wave number for thermally insulated and impermeable boundaries.

but their corresponding values are different in magnitude. Figure 6 exhibits the variation of attenuation with wave number $\xi$. The values of attenuation coefficients for smaller values of wave number increase monotonically and become oscillatory for all the cases in $4 \leq \xi \leq 6$, which come to be dispersionless for higher values of $\xi$. Figure 7 exhibits the variation of the normal velocity component with wave number $\xi$. It is noticed that due to imperfect boundary the normal velocity component for LSH10WC shows a good difference in the magnitude value while compared with 


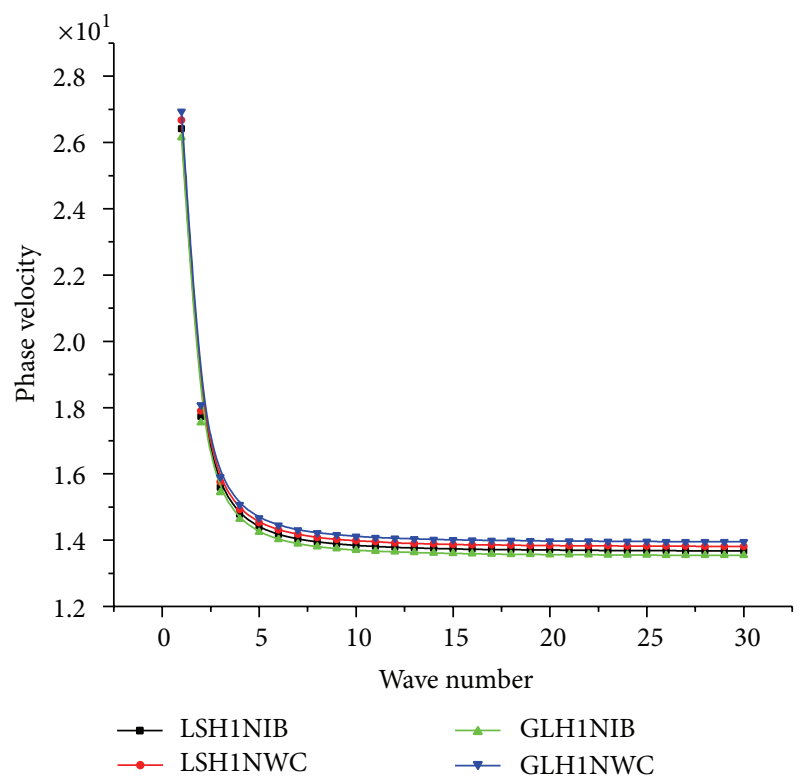

FIGURE 5: Variation of phase velocity with respect to wave number for isothermal and isoconcentrated boundaries.

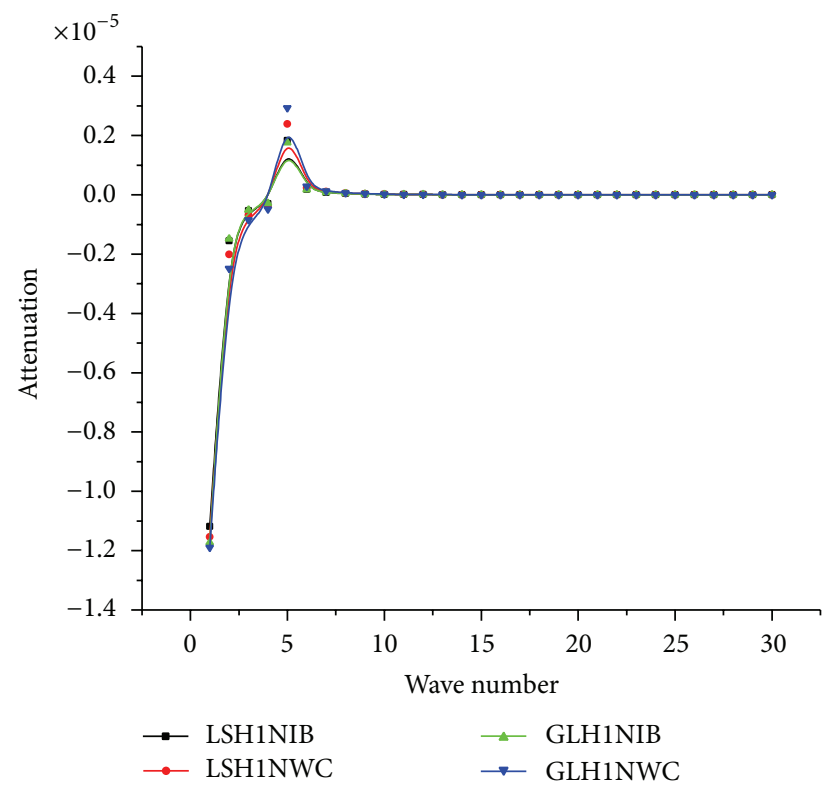

FIGURE 6: Variation of attenuation with respect to wave number for isothermal and isoconcentrated boundaries.

LSH10IB, though in both the cases the values get a sudden fall for $18 \leq \xi \leq 30$. On the other hand, a worthy difference in the behavior of curves is noticed when compared with the curves for thermally insulated and impermeable boundaries, as shown in Figure 4.

\section{Conclusion}

The propagation of Rayleigh type surface waves at an imperfect boundary between an inviscid liquid layer and a homogeneous isotropic microstretch thermoelastic diffusion solid half-space subjected to thermally insulated/isothermal and

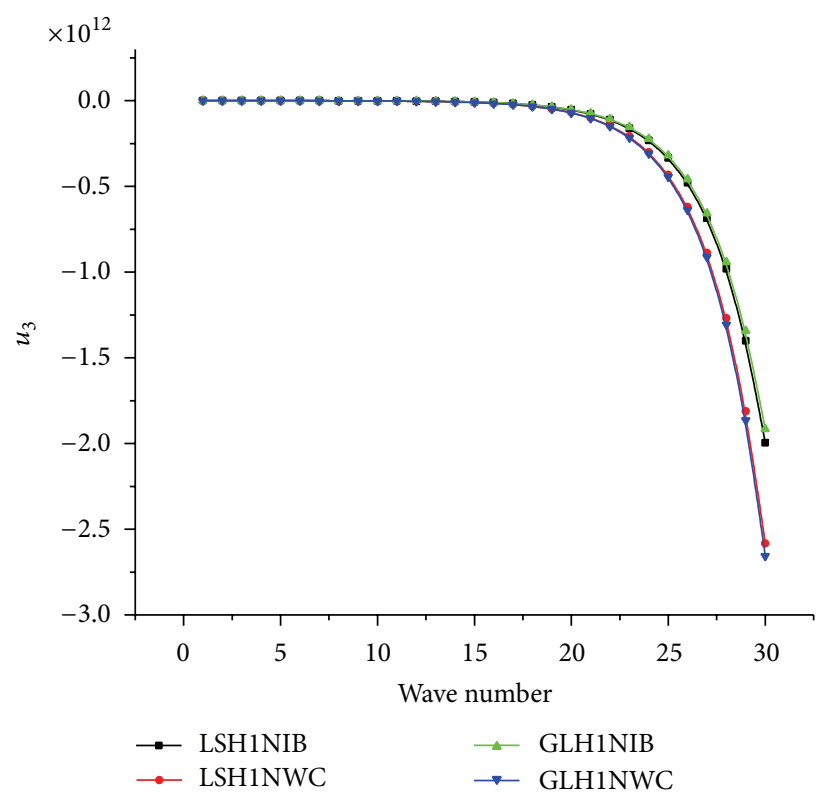

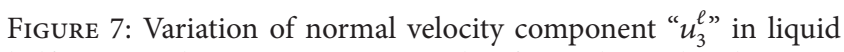
half-space with respect to wave number for isothermal and isoconcentrated boundaries.

impermeable/isoconcentrated boundaries has been investigated. A secular equation for surface wave propagation in the considered medium is derived. A significant effect of an imperfect boundary along with the relaxation times on the phase velocity, attenuation coefficient, and the normal velocity component for the considered mathematical model has been observed. Effect due to imperfect boundary along with relaxation times for LSH10IB and LSH10WC is not much significant as compared with the case of thermally insulated and impermeable boundaries. In case of GLHINIB and GLH1NWC, the attenuation coefficients have very good impact on imperfect boundary when compared to GLH10IB and GLH10WC, respectively, for the smaller value of wave number. It is perceived that the trend of variations and behavior of the all resulting quantities try to converge toward a particular value with the increase of wave number, except for $u_{3}^{\ell}$ in case of GLH10IB at the insulated/isothermal boundary, which shows a significant difference due to imperfect boundary. Among all the graphs, the normal velocity components for both thermally insulated/isothermal and impermeable/isoconcentrated boundaries show respectable variance due to imperfect boundary.

\section{Appendices}

A.

Consider

$$
\begin{gathered}
A_{1}^{*}=\frac{d_{12}}{d_{11}}, \quad B_{1}^{*}=\frac{d_{13}}{d_{11}}, \quad C_{1}^{*}=\frac{d_{14}}{d_{11}}, \quad D_{1}^{*}=\frac{d_{15}}{d_{11}}, \\
A_{2}^{*}=\frac{\left(a_{4} b_{26}+b_{27}\left(1-\delta^{2}\right)+a_{1} a_{5}\right)}{a_{4}\left(1-\delta^{2}\right)},
\end{gathered}
$$




$$
\begin{aligned}
& B_{2}^{*}=\frac{\left(b_{26} b_{27}-a_{1} a_{5} \xi^{2}\right)}{a_{4}\left(1-\delta^{2}\right)}, \\
& d_{11}=-\left(l_{11}+b_{13} l_{18}\right) \text {, } \\
& d_{12}=-l_{12}+b_{11} l_{11}-b_{12} l_{15}+b_{13}\left(\xi^{2} l_{18}-l_{19}\right)-a_{2} l_{22} \text {, } \\
& d_{13}=-l_{13}+b_{11} l_{12}+b_{12}\left(\xi^{2} l_{15}-l_{16}\right) \\
& +b_{13}\left(\xi^{2} l_{19}-l_{20}\right)-a_{2} \xi^{2} l_{22}-a_{2} l_{23} \\
& d_{14}=-l_{14}+b_{11} l_{13}+b_{12}\left(\xi^{2} l_{16}-l_{17}\right) \\
& +b_{13}\left(\xi^{2} l_{20}-l_{21}\right)+a_{2} l_{23} \\
& d_{15}=b_{11} l_{14}+b_{12} \xi^{2} l_{17} \\
& +b_{13} \xi^{2} l_{21}+a_{2} \xi^{2} l_{24}-a_{2} l_{24} \\
& l_{11}=b_{23} \delta_{1}^{2}, \quad l_{12}=\delta_{1}^{2}\left(b_{19} b_{22}-g_{12}\right)+b_{23} g_{14}, \\
& l_{13}=-b_{22} \xi^{2}\left(b_{19} \delta_{1}^{2}+g_{11}\right)-g_{12} g_{14} \\
& +b_{23} g_{13}+a_{21}\left(g_{15}-b_{15}\right) \text {, } \\
& l_{14}=\xi^{2}\left(b_{22} g_{11}-a_{2} b_{15}\right)-g_{12} g_{13}-a_{21} \xi^{2}\left(g_{15}+b_{15}\right) \text {, } \\
& l_{15}=\delta_{1}^{2}\left(a_{14} b_{19}+b_{17} b_{23}\right), \\
& l_{16}=\delta_{1}^{2}\left(g_{12} b_{17}-a_{14} b_{19} \xi^{2}\right)-a_{14} g_{16}+g_{18} b_{23}+a_{21} g_{19} \text {, } \\
& l_{17}=\xi^{2}\left(a_{14} g_{16}-a_{21} g_{19}\right)-g_{12} g_{18}, \\
& l_{18}=-a_{14} \delta_{1}^{2} \\
& l_{19}=\delta_{1}^{2}\left(a_{14} \xi^{2}-b_{17} b_{22}\right)-a_{14} g_{14}, \\
& l_{20}=\xi^{2}\left(b_{17} b_{22} \delta_{1}^{2}+a_{14} g_{14}\right) \\
& -a_{14} g_{13}+b_{22} g_{18}+a_{21} g_{20} \\
& l_{21}=\xi^{2}\left(a_{14} g_{13}-b_{22} g_{18}-a_{21} g_{20}\right) \text {, } \\
& l_{22}=a_{8} b_{22}-a_{14} b_{15}, \\
& l_{23}=a_{14}\left(\xi^{2} b_{15}-g_{21}\right)+b_{22} g_{22}-a_{8} g_{23}-b_{22} g_{24} \text {, } \\
& l_{24}=\xi^{2}\left(a_{14} g_{21}-b_{22} g_{22}\right)+g_{23} g_{24} \text {, } \\
& g_{11}=b_{15} b_{20}-b_{16} b_{19}, \quad g_{12}=b_{23} \xi^{2}-b_{24} \text {, } \\
& g_{13}=b_{14} b_{20}-b_{16} b_{18}, \quad g_{14}=b_{16}-b_{18} \delta_{1}^{2} \text {, } \\
& g_{15}=b_{14} b_{19}-b_{15} b_{18}, \quad g_{16}=b_{15} b_{20}-b_{16} b_{19}, \\
& g_{18}=a_{8} b_{20}-b_{16} b_{17}, \quad g_{19}=a_{8} b_{19}-b_{15} b_{17}, \\
& g_{20}=a_{8} b_{18}-b_{14} b_{17}, \quad g_{21}=b_{14} b_{19}-b_{15} b_{18} \text {, } \\
& g_{22}=a_{8} b_{19}-b_{15} b_{17}, \quad g_{23}=\left(b_{22} \xi^{2}-b_{24}\right), \\
& g_{24}=a_{8} b_{18}-b_{14} b_{17}
\end{aligned}
$$

B.

Consider

$$
\begin{aligned}
& n_{1 p}=-\left[-l_{22} m_{p}^{6}+\left(\xi^{2} l_{22}-l_{23}\right) m_{p}^{4}\right. \\
& \left.+\left(\xi^{2} l_{23}-l_{24}\right) m_{p}^{2}+\xi^{2} l_{24}\right] \\
& \times\left[l_{11} m_{p}^{6}+l_{12} m_{p}^{4}+l_{13} m_{p}^{2}+l_{14}\right]^{-1}, \\
& n_{2 p}=\left[-l_{15} m_{p}^{6}+\left(\xi^{2} l_{15}-l_{16}\right) m_{p}^{4}\right. \\
& \left.+\left(\xi^{2} l_{16}-l_{17}\right) m_{p}^{2}+\xi^{2} l_{17}\right] \\
& \times\left[l_{11} m_{p}^{6}+l_{12} m_{p}^{4}+l_{13} m_{p}^{2}+l_{14}\right]^{-1}, \\
& n_{3 p}=-\left[-l_{18} m_{p}^{8}+\left(\xi^{2} l_{18}-l_{19}\right) m_{p}^{6}+\left(\xi^{2} l_{19}-l_{20}\right) m_{p}^{4}\right. \\
& \left.+\left(\xi^{2} l_{20}-l_{21}\right) m_{p}^{2}+\xi^{2} l_{21}\right] \\
& \times\left[l_{11} m_{p}^{6}+l_{12} m_{p}^{4}+l_{13} m_{p}^{2}+l_{14}\right]^{-1}, \\
& \text { for }(p=1,2,3,4) \text {, } \\
& n_{4 p}=\frac{a_{5}\left(m_{p}^{2}-\xi^{2}\right)}{\left(b_{27}+a_{4} m_{p}^{2}\right)}, \quad \text { for }(p=5,6) \text {, } \\
& \Delta_{12}=-\frac{\Delta_{1}}{\Delta_{2}} \\
& \Delta_{2}=\left|\begin{array}{lllllll}
k_{21} & k_{22} & k_{23} & k_{24} & k_{25} & k_{26} & k_{27} \\
k_{31} & k_{32} & k_{33} & k_{34} & k_{35} & k_{36} & 0 \\
k_{41} & k_{42} & k_{43} & k_{44} & k_{45} & k_{46} & 0 \\
k_{51} & k_{52} & k_{53} & k_{54} & k_{55} & k_{56} & 0 \\
k_{61} & k_{62} & k_{63} & k_{64} & k_{65} & k_{66} & 0 \\
k_{71} & k_{72} & k_{73} & k_{74} & 0 & 0 & 0 \\
k_{81} & k_{82} & k_{83} & k_{84} & 0 & 0 & 0
\end{array}\right|_{7 \times 7},
\end{aligned}
$$




$$
\begin{gathered}
\Delta_{3}=\left|\begin{array}{llllll}
k_{21} & k_{22} & k_{23} & k_{25} & k_{26} & 0 \\
k_{31} & k_{32} & k_{33} & k_{35} & k_{36} & k_{38} \\
k_{41} & k_{42} & k_{43} & k_{45} & k_{46} & 0 \\
0 & 0 & 0 & k_{55} & k_{56} & 0 \\
k_{71} & k_{72} & k_{73} & 0 & 0 & 0 \\
k_{81} & k_{82} & k_{83} & 0 & 0 & 0
\end{array}\right|_{6 \times 6} \\
\Delta_{4}=\left|\begin{array}{llllll}
k_{21} & k_{22} & k_{23} & k_{25} & k_{26} & k_{27} \\
k_{31} & k_{32} & k_{33} & k_{35} & k_{36} & 0 \\
k_{41} & k_{42} & k_{43} & k_{45} & k_{46} & 0 \\
0 & 0 & 0 & k_{55} & k_{56} & 0 \\
k_{71} & k_{72} & k_{73} & 0 & 0 & 0 \\
k_{81} & k_{82} & k_{83} & 0 & 0 & 0
\end{array}\right|_{6 \times 6} \\
\Delta_{5}=\left|\begin{array}{llllll}
k_{21} & k_{22} & k_{25} & k_{26} & 0 \\
k_{31} & k_{32} & k_{35} & k_{36} & k_{38} \\
k_{41} & k_{42} & k_{45} & k_{46} & 0 \\
k_{71} & k_{72} & k_{75} & 0 & 0 \\
k_{81} & k_{82} & k_{85} & 0 & 0
\end{array}\right|_{5 \times 5}, \\
\Delta_{6}=\left|\begin{array}{llllll}
k_{21} & k_{22} & k_{25} & k_{26} & k_{27} \\
k_{31} & k_{32} & k_{35} & k_{36} & 0 \\
k_{41} & k_{42} & k_{45} & k_{46} & 0 \\
k_{71} & k_{72} & k_{75} & 0 & 0 \\
k_{81} & k_{82} & k_{85} & 0 & 0
\end{array}\right|_{5 \times 5}
\end{gathered}
$$

\section{Conflict of Interests}

The authors declare that there is no conflict of interests regarding the publication of this paper.

\section{References}

[1] A. C. Eringen, "Linear theory of micropolar elasticity", Journal of Mathematics and Mechanics, vol. 15, pp. 909-923, 1966.

[2] A. C. Eringen, "Theory of micropolar fluids," Journal of Mathematics and Mechanics, vol. 15, pp. 1-18, 1966.

[3] A. C. Eringen, "Foundation of micropolar thermoelasticity," Course of Lectures 23, CISM, Springer, Udine, Italy, 1970.

[4] W. Nowacki, "Couple-stresses in the theory of thermoelasticity," in Irreversible Aspects of Continuum Mechanics and Transfer of Physical Characteristics in Moving Fluids: Symposia Vienna, June 22-28, 1966, IUTAM Symposia, pp. 259-278, 1968.

[5] D. S. Chandrasekharaiah, "Heat-flux dependent micropolar thermoelasticity," International Journal of Engineering Science, vol. 24, no. 8, pp. 1389-1395, 1986.

[6] A. C. Eringen, "Micropolar elastic solids with stretch," Ari Kitabevi Matbaasi, vol. 24, pp. 1-18, 1971.

[7] A. C. Eringen, "Theory of thermomicrostretch elastic solids," International Journal of Engineering Science, vol. 28, no. 12, pp. 1291-1301, 1990.

[8] A. C. Eringen, Microcontinuum Field Theories: I. Foundations and Solids, Springer, New York, NY, USA, 1999.

[9] W. Nowacki, "Dynamical problems of thermodiffusion in solids-I," Bulletin of Polish Academy of Sciences Series, Science and Technology, vol. 22, pp. 55-64, 1974.

[10] W. Nowacki, "Dynamical problems of thermodiffusion in solids-II," Bulletin of Polish Academy of Sciences Series, Science and Technology, vol. 22, pp. 129-135, 1974.
[11] W. Nowacki, "Dynamical problem of thermodiffusion in solids. III," Bulletin of Polish Academy of Sciences Series, Science and Technology, vol. 22, pp. 275-276, 1974.

[12] W. Nowacki, "Dynamic problems of diffusion in solids," Engineering Fracture Mechanics, vol. 8, no. 1, pp. 261-266, 1976.

[13] W. Dudziak and S. J. Kowalski, "Theory of thermodiffusion for solids," International Journal of Heat and Mass Transfer, vol. 32, no. 11, pp. 2005-2013, 1989.

[14] Z. S. Olesiak and Y. A. Pyryev, "A coupled quasi-stationary problem of thermodiffusion for an elastic cylinder," International Journal of Engineering Science, vol. 33, no. 6, pp. 773-780, 1995.

[15] H. H. Sherief, F. A. Hamza, and H. A. Saleh, "The theory of generalized thermoelastic diffusion," International Journal of Engineering Science, vol. 42, no. 5-6, pp. 591-608, 2004.

[16] R. Kumar and T. Kansal, "Propagation of Lamb waves in transversely isotropic thermoelastic diffusive plate," International Journal of Solids and Structures, vol. 45, no. 22-23, pp. 58905913, 2008.

[17] J. Wu and Z. Zhu, “The propagation of Lamb waves in a plate bordered with layers of a liquid," Journal of the Acoustical Society of America, vol. 91, no. 2, pp. 861-867, 1992.

[18] Z. Zhu and J. Wu, "The propagation of Lamb waves in a plate bordered with a viscous liquid," Journal of the Acoustical Society of America, vol. 98, no. 2I, pp. 1057-1064, 1995.

[19] R. Quintanilla, "On the spatial decay for the dynamical problem of thermo-microstretch elastic solids," International Journal of Engineering Science, vol. 40, no. 2, pp. 109-121, 2002.

[20] R. Kumar and G. Partap, "Free vibration analysis of waves in a microstretch elastic plate," Tamkang Journal of Science and Engineering, vol. 13, no. 3, pp. 227-233, 2010.

[21] I. A. Abbas and M. I. A. Othman, "Plane waves in generalized thermo-microstretch elastic solid with thermal relaxation using finite element method," International Journal of Thermophysics, vol. 33, pp. 2407-2423, 2012.

[22] M. I. A. Othman, S. Y. Atwa, A. Jahangir, and A. Khan, "Effect of magnetic field and rotation on generalized thermomicrostretch. Elastic solid with mode-I crack under the Green Naghdi theory," Computational Mathematics and Modeling, vol. 24, no. 4, pp. 566-591, 2013.

[23] J. N. Sharma, S. Kumar, and Y. D. Sharma, "Propagation of rayleigh surface waves in microstretch thermoelastic continua under inviscid fluid loadings," Journal of Thermal Stresses, vol. 31, no. 1, pp. 18-39, 2008.

[24] R. Kumar and G. Partap, "Wave propagation in microstretch thermoelastic plate bordered with layers of inviscid liquid," Multidiscipline Modeling in Materials and Structures, vol. 5, no. 2, pp. 171-184, 2009.

[25] R. Kumar, S. Ahuja, and S. K. Garg, "Rayleigh waves in isotropic microstretch thermoelastic diffusion solid half space," Latin American Journal of Solids and Structures, vol. 11, no. 2, pp. 299319, 2014.

[26] J. D. Achenbach, Wave Propagation in Elastic Solids, NorthHolland, Amsterdam, The Netherlands, 1976.

[27] A. C. Eringen, "Plane waves in nonlocal micropolar elasticity," International Journal of Engineering Science, vol. 22, no. 8-10, pp. 1113-1121, 1984. 

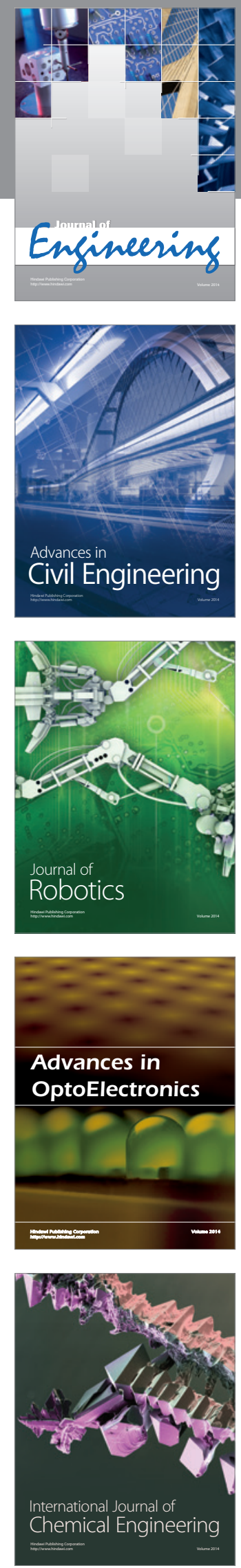

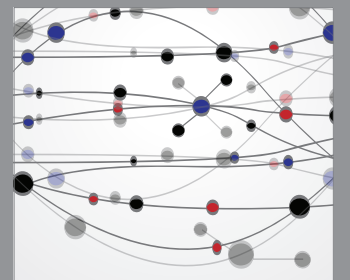

The Scientific World Journal
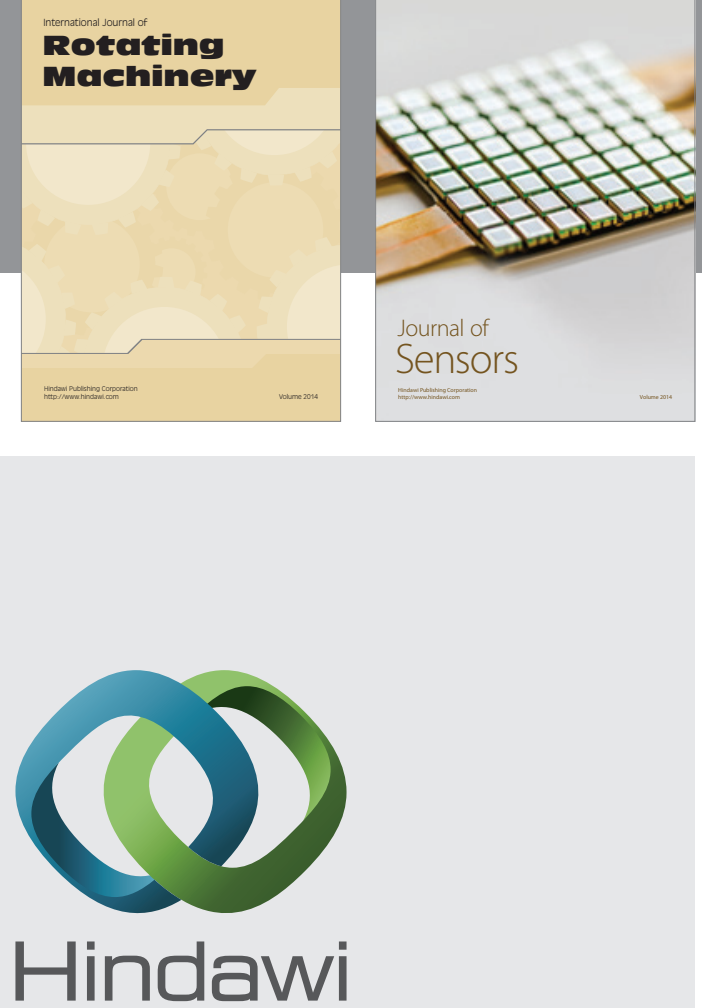

Submit your manuscripts at http://www.hindawi.com
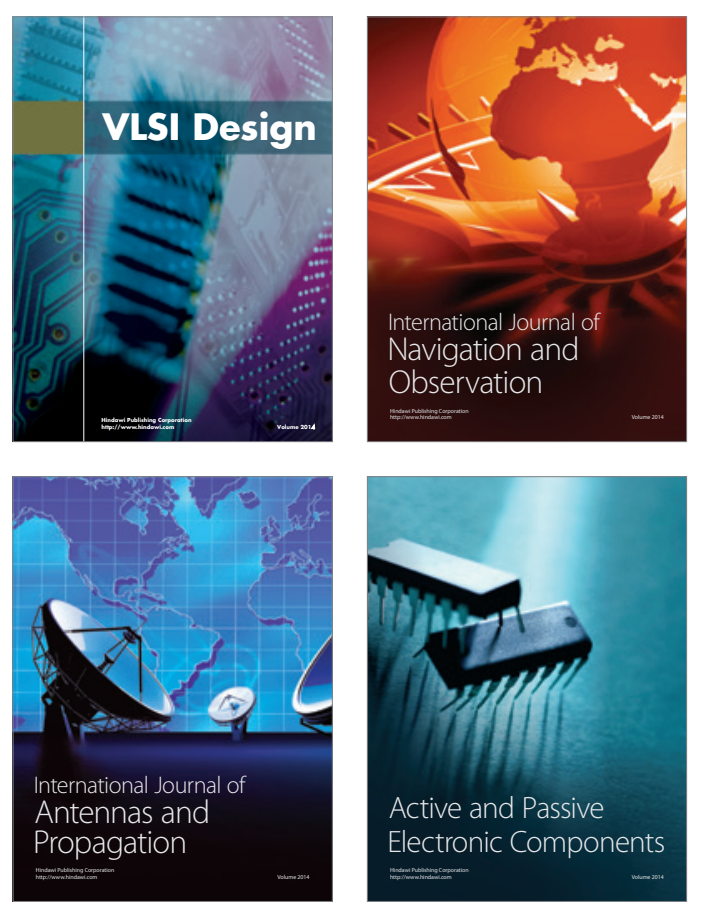
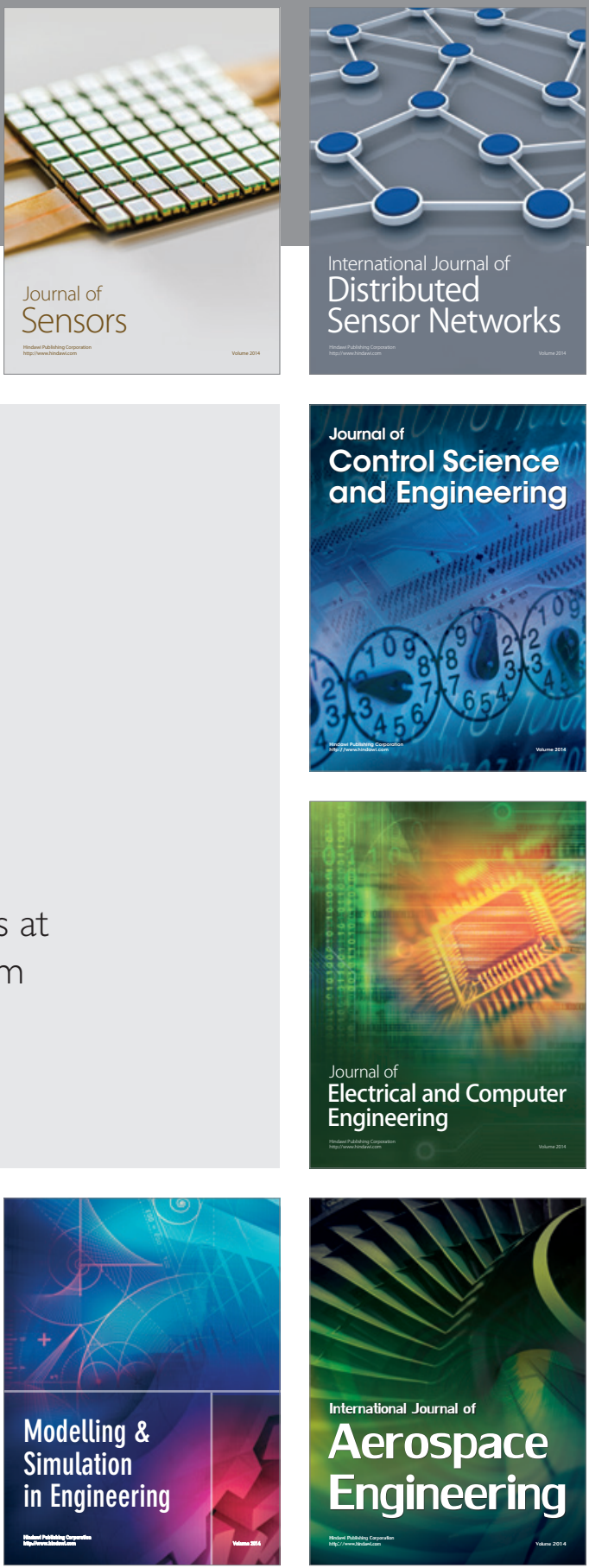

Journal of

Control Science

and Engineering
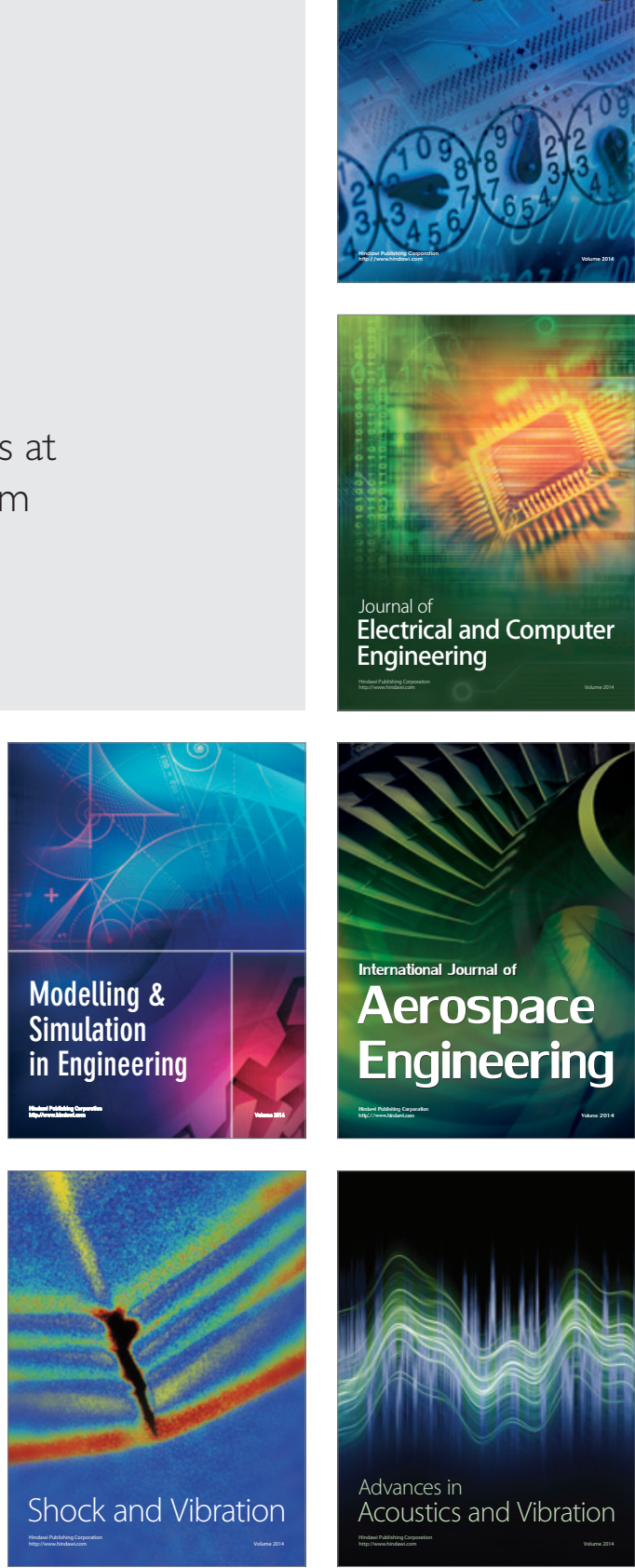